\title{
Unprecedented Piezoresistance Coefficient in Strained Silicon Carbide
}

\author{
Junfeng Cui, ${ }^{\dagger \dagger}$ Zhenyu Zhang, ${ }^{*}{ }^{\dagger}, \quad$ Dongdong Liu, ${ }^{\dagger, \dagger}$ Danli Zhang, ${ }^{\S}$ Wei Hu, ${ }^{\dagger}$ Li Zou, ${ }^{\|}$Yao Lu, ${ }^{\perp}$ \\ Chi Zhang, ${ }^{\#}$ Huanhuan Lu, ${ }^{\S}$ Chun Tang, ${ }^{*}, \mathbb{I}$ Nan Jiang, ${ }^{*}{ }^{*}$ Ivan P. Parkin, ${ }^{\nabla}$ and Dongming Guo ${ }^{\dagger}$
}

${ }^{\dagger}$ Key Laboratory for Precision and Non-Traditional Machining Technology of Ministry of Education and "School of Naval Architecture, State Key Laboratory of Structural Analysis for Industrial Equipment, Dalian University of Technology, Dalian 116024, China

${ }^{\ddagger}$ Key Laboratory of Marine Materials and Related Technologies, Ningbo Institute of Materials Technology and Engineering, Chinese Academy of Sciences, Ningbo 315201, China

${ }^{\S}$ Center for Advancing Materials Performance from the Nanoscale (CAMP-Nano) \& Hysitron Applied Research Center in China (HARCC), State Key Laboratory for Mechanical Behavior of Materials, Xi'an Jiaotong University, Xi'an 710049, China

${ }^{\perp}$ Department of Chemistry, School of Biological and Chemical Sciences, Queen Mary University of London, London E1 4NS, U.K.

${ }^{\#}$ Beijing Institute of Nanoenergy and Nanosystems, Chinese Academy of Sciences, Beijing 100083, China

${ }^{\mathbb{T}}$ Faculty of Civil Engineering and Mechanics, Jiangsu University, Zhenjiang 212013, China

${ }^{\nabla}$ Materials Chemistry Research Centre, Department of Chemistry, University College London, 20 Gordon Street, London WC1H OAJ, U.K.

\begin{abstract}
Reports reveal that the piezoresistance coefficients of silicon carbide ( $\mathrm{SiC}$ ) nanowires (NWs) are 2 to 4 times smaller than those of their corresponding bulk counterparts. It is a challenge to eliminate contamination in adhering NWs onto substrates. In this study, a new setup was developed, in which NWs were manipulated and fixed by a goat hair and conductive silver epoxy in air, respectively, in the absence of any depositions. The goat hair was not consumed during manipulation of the NWs. The process took advantage of the stiffness and tapered tip of the goat hair, which is unlike the loss
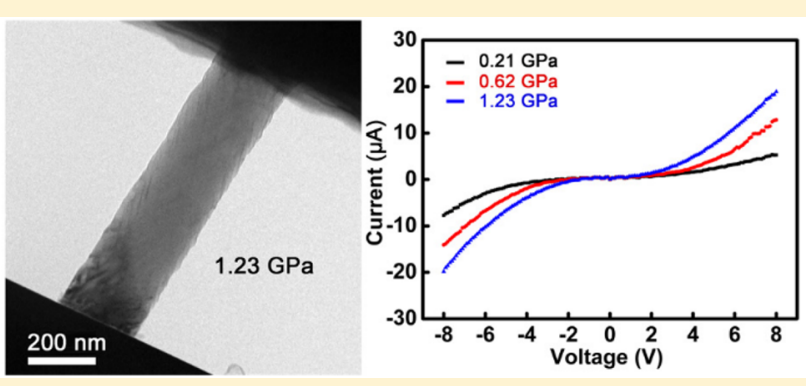
issue of beam sources in depositions. With the new fixing method, in situ transmission electron microscopy (TEM) electromechanical coupling measurements were performed on pristine SiC NWs. The piezoresistance coefficient and carrier mobility of $\mathrm{SiC} \mathrm{NW}$ are $-94.78 \times 10^{-11} \mathrm{~Pa}^{-1}$ and $30.05 \mathrm{~cm}^{2} \mathrm{~V}^{-1}$ $\mathrm{s}^{-1}$, respectively, which are 82 and 527 times respectively greater than those of $\mathrm{SiC}$ NWs reported previously. We, for the first time, report that the piezoresistance coefficient of $\mathrm{SiC} \mathrm{NW}$ is 17 times those of its bulk counterparts. These findings provide new insights to develop high performance $\mathrm{SiC}$ devices and to help avoid catastrophic failure when working in harsh environments.
\end{abstract}

KEYWORDS: Piezoresistance coefficient, strain, in situ TEM, electromechanical coupling, SiC

Ctrain engineering is considered as one of the most $\checkmark$ promising strategies for developing high performance devices at the nanoscale. ${ }^{1,2}$ It has been widely used to explore altering elementary properties such as band structure, to enhance the performance of a device. ${ }^{3}$ In fact, theory predicts that strain has a large effect on the band structure of nanowires (NWs), which in turn has an influence on their physical properties. Nanoscale devices aim to in part decrease the critical dimension to improve performance while reducing power consumption. ${ }^{4}$ Individual stressed semiconductor NWs have been demonstrated as an ideal material for exploring the strain-related electronic properties of devices. ${ }^{5,6}$ The piezoresistance coefficient of silicon ( $\mathrm{Si}$ ) NWs is 37.8 times that of bulk $\mathrm{Si}^{2}{ }^{2}$ This giant piezoresistance effect in strained NWs has significant implications in NW-based flexible electronics as well as in nanoelectromechanical system (NEMS). The piezoresistance effect converts elusive mechanical signals to easily accessible electrical signals at the nanoscale, enabling NWs to function as key building blocks of nanoscale devices. ${ }^{7}$ Nevertheless, Si has a low energy gap of $1.12 \mathrm{eV}$ and is typically used at less than $200{ }^{\circ} \mathrm{C}$ because of the limitations of its physical and chemical properties. ${ }^{8}$ 


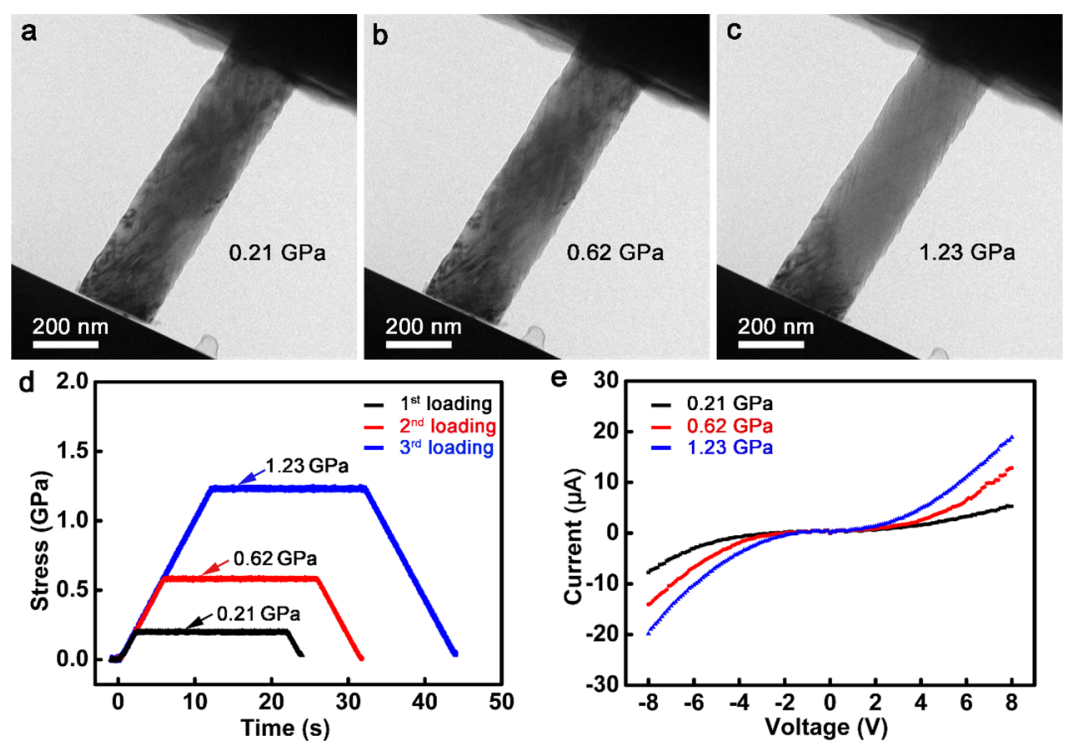

Figure 1. TEM images of an NW under axially compressive stress at (a) 0.21 , (b) 0.62, and (c) $1.23 \mathrm{GPa}$; (d) stress and (e) current as a function of time and voltage, respectively, at three different axially compressive stresses.

Silicon carbide $(\mathrm{SiC})$ has exceptional physical and mechanical properties, consisting of low density, high strength, high thermal conductivity, stability at high temperature, high resistance to shocks, low thermal expansion, high refractive index, good biocompatibility, wide bandgap, and chemical inertness. ${ }^{9}$ These properties make $\mathrm{SiC}$ suitable for use at high temperature, ${ }^{10,11}$ high power, ${ }^{12}$ and high frequency as well as in harsh environments. ${ }^{13-15} \mathrm{SiC}$ has become one of the most promising materials for power electronics, heterogeneous catalyst supports, wear resistance devices, and biomaterials.' Fabrication methods originating from $\mathrm{Si}$ technology have been applied to $\mathrm{SiC}$. As a result, $\mathrm{SiC}$ is widely used in electronic, optoelectronic, and electromechanical devices as well as substrates for growing high performance graphene. ${ }^{16-18}$ Double toughness is obtained in a $\mathrm{SiC}$ whisker-reinforced matrix through adding a small fraction of $\mathrm{SiC}$ NWs. SiC nanoresonators have higher frequencies than gallium arsenide (GaAs) and Si counterparts for a given dimension. ${ }^{12}$ A higher piezoresistance coefficient is expected in $\mathrm{SiC} \mathrm{NWs}$ as compared with bulk counterparts, on account of the increased surface-to-volume ratio. Nonetheless, the piezoresistance coefficients of $\mathrm{SiC} \mathrm{NWs}^{19,20}$ are 2 to 4 times smaller than those of bulk counterparts. Thus, there are no reports on the higher piezoresistance coefficient in pristine $\mathrm{SiC}$ NWs as compared to bulk counterparts. ${ }^{8}$

There are three main effects on the measurement of physical properties of NWs, relating to size-strengthening, sizeindependence, and size-weakening. The tremendous difference among measurement results is attributed to the intrinsic limitations of various testing protocols. ${ }^{21}$ To evaluate the piezoresistance effect of $\mathrm{SiC}$ NWs, electromechanical coupling measurements are required, in which mechanical manipulations and electrical measurements should be conducted simultaneously. Conventionally, electrochemical coupling measurements are carried out by interfacing the NWs with lithographically fabricated electrodes. ${ }^{2}$ This method inevitably has stability and reliability issues when subjected to mechanical forces. To solve this problem, electron beam deposition (EBD) ${ }^{20,22-24}$ and focused ion beam (FIB) deposition ${ }^{21,25}$ are employed to interface the NWs on samples. However, EBD and FIB depositions will induce contamination and damage on the NWs. Electromechanical coupling measurements are sensitive to contamination and damage of the NWs, resulting in considerable discrepancy of measurements between deposited and pristine NWs. It is a challenge to eliminate the contamination and damage induced by such deposition methods.

In this study, a new setup was developed, where NWs were manipulated and fixed by a goat hair and conductive silver (Ag) epoxy, respectively. With this method, a new metrology was performed on pristine NWs by in situ transmission electron microscopy (TEM) electromechanical coupling measurements. An unprecedented piezoresistance effect was observed, and the fundamental mechanism was elucidated by ab initio simulations.

Results and Discussion. Figure S1 illustrates the scanning electron microscopy (SEM) and TEM images as well as electron energy dispersive spectroscopy (EDS) and Raman spectra of 3C-SiC NWs. Diameters of NWs vary from tens of to hundreds of nanometers, as illustrated in Figure S1(a). The selected area electron diffraction (SAED) pattern exhibits monocrystalline characteristics (Figure $S 1(b)$ ), which is in good agreement with HRTEM images in Figures S1(c,d). The axial direction of the NW is along <111> orientation, as confirmed by the HRTEM image in Figure $\mathrm{S} 1(\mathrm{c}) . \mathrm{Si}$ and carbon elements are found in Figure S1(e), and there were no other elements observed. This reveals that the SiC NWs are pristine without doping elements. Raman spectra in Figure S1(f) with peaks at 796 and $970 \mathrm{~cm}^{-1}$ are consistent with those of single crystal $3 \mathrm{C}-\mathrm{SiC}$ NWs. ${ }^{12}$

Figure S2(a) depicts the schematic diagram of the in situ TEM electromechanical measurement. The diameter and length of the NW in Figure S2(b) are 249 and $968 \mathrm{~nm}$, respectively. An axially compressive force was applied by a flat tungsten (W) tip. During compression, a voltage was exerted to the NW, and the compressing current was simultaneously measured. In this process, in situ TEM measurements were performed to observe the variation of nanostructure under axially compressive stress. With this method, in situ TEM electromechanical measurement is conducted. The NW was 

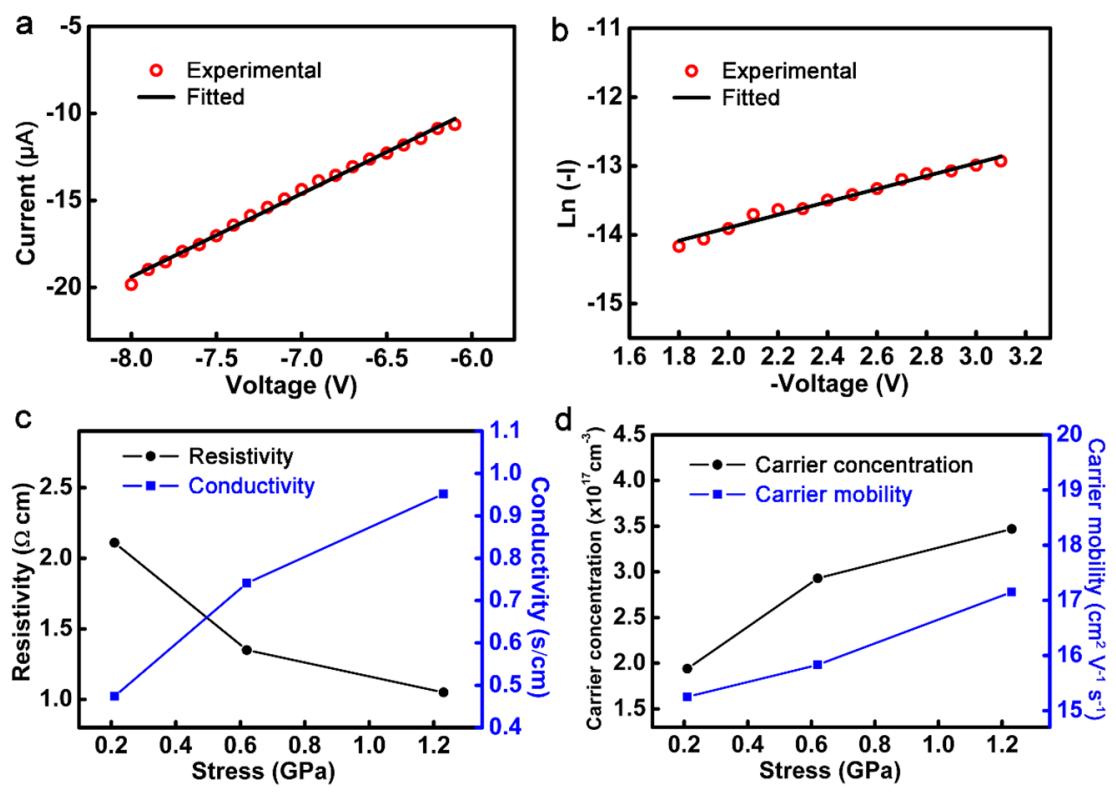

Figure 2. Experimental and fitted data of (a) current and (b) $\ln (-I)$ as a function of voltage at large and intermediate bias regimes, respectively, in Figure 3(e) at $1.23 \mathrm{GPa}$; (c) resistivity and conductivity and (d) carrier concentration and carrier mobility as a function of stress.

fixed by conductive Ag epoxy, absent of depositions, eliminating the contamination and damage induced by conventional depositions. Hence, an in situ TEM electromechanical measurement was developed to measure the piezoresistance effect of NWs.

Figure $1(\mathrm{a}-\mathrm{c})$ shows the TEM images of an NW under axially compressive stress at $0.21,0.62$, and $1.23 \mathrm{GPa}$, respectively. Figure $1(\mathrm{~d})$ shows the stress as a function of time at three different axially compressive stresses. In situ dynamic TEM electromechanical measurements of an NW at 10,30 , and $60 \mu \mathrm{N}$ are played in Movies S1, S2, and S3, respectively. Current-voltage $(I-V)$ curves in Figure 1(e) exhibit symmetrically nonlinear characteristics, indicating metal-semiconductor-metal $(\mathrm{M}-\mathrm{S}-\mathrm{M})$ contact in $\mathrm{W}-$ $\mathrm{SiC}-\mathrm{Ag}$ connections. The nonlinearity of $I-V$ curves is because of the Schottky barrier between a metal and semiconductor. $^{26-29}$ This is different from the ohmic contact induced by contaminations and damages of $\mathrm{EBD}^{20,22-24}$ and $\mathrm{FIB}^{21,25}$ depositions. In our work, a new setup was developed, in which NWs were manipulated and fixed by a goat hair and conductive Ag epoxy, respectively. Using the goat hair to manipulate NWs does not require depositions, and contaminations and damages are effectively eliminated. NWs were picked up by the goat hair tip in the developed setup. This function uses the stiffness and tapered tip of the goat hair, and the goat hair has no consumption during the operation on NWs, which is unlike the loss issue of beam sources taken place in depositions.

Figure 1(e) shows that the greater axially compressive stress leads to higher current and better electrical conductivity. According to the thermionic emission theory of the $\mathrm{M}-\mathrm{S}-\mathrm{M}$ structure, the $I-V$ curve is linear at the large bias regime. Resistance of an NW is defined ${ }^{26,27}$

$$
R \approx \frac{\mathrm{d} V}{\mathrm{dI}}
$$

where $R$ is the resistance, and $\mathrm{d} V$ and $\mathrm{d} I$ are the differentials of the voltage and current, respectively. Figure 2(a) pictures the experimental and fitted data under a large bias regime of 1.23
GPa. The $R$ of the NW in Figure 1(e) was calculated at 209.1 $\mathrm{k} \Omega$ at $1.23 \mathrm{GPa}$. Resistivity, $\rho$, is presented

$$
\rho=\frac{R A}{L}
$$

where $A$ and $L$ are the area and length of an NW, respectively. According to eq 2, increasing the compressive stress in Figure $1(\mathrm{e})$, the area of the NW increases and the length decreases. If the resistance is invariable, resistivity increases, resulting, in a reverse manner, the decrease of resistance and increase of current. Conductivity, $C$, is calculated

$$
C=\frac{1}{\rho}
$$

$\rho$ and $C$ are $1.05 \Omega \cdot \mathrm{cm}$ and $0.95 \mathrm{~s} / \mathrm{cm}$, respectively. In Figures 1(e) and 2(b), a logarithmic plot of current $I$ as a function of bias $V$ gives approximately a straight line of slope, $S^{19,26,27}$

$$
S=\frac{q}{k T}-\frac{1}{E_{0}}
$$

where $q$ is the elemental charge, $k$ is the Boltzmann constant, $T$ is the temperature in Kelvin, and $E_{0}$ is a parameter that depends on the carrier density. $E_{0}$ is given ${ }^{26}$

$$
E_{0}=E_{00} \operatorname{coth}\left(\frac{q E_{00}}{k T}\right)
$$

where $E_{00}$ is a parameter depending on the carrier density. $E_{00}$ is obtained, ${ }^{19,26,27}$

$$
E_{00}=\frac{\hbar}{2}\left(\frac{N_{\mathrm{d}}}{m^{*} \varepsilon}\right)^{1 / 2}=\frac{\hbar}{2}\left(\frac{N_{\mathrm{d}}}{m_{\mathrm{r}} m \varepsilon_{\mathrm{r}} \varepsilon_{0}}\right)^{1 / 2}=18.5 \cdot 10^{-15}\left(\frac{N_{\mathrm{d}}}{m_{\mathrm{r}} \varepsilon_{\mathrm{r}}}\right)^{1 / 2} \mathrm{eV}
$$

where $\hbar$ is the Planck constant divided by $2 \pi, N_{\mathrm{d}}$ is the donor density between the metal and semiconductor interface, $m^{*}$ $\left(=m_{\mathrm{r}} m\right)$ is the effective mass of an electron, $\varepsilon\left(=\varepsilon_{\mathrm{r}} \varepsilon_{0}\right)$ is the permittivity of an NW, $m_{\mathrm{r}}$ is the relative mass of an electron, $m$ is the free-electron mass, $\varepsilon_{\mathrm{r}}$ is the relative permittivity of an 

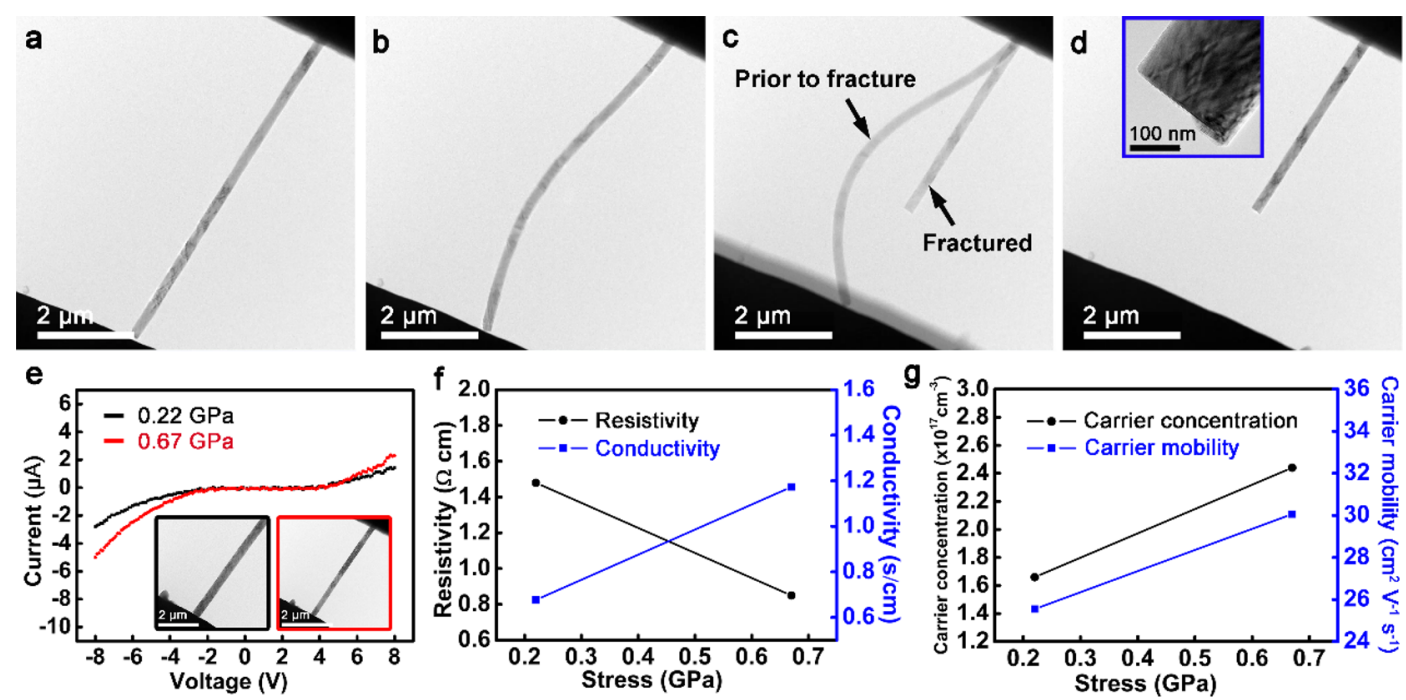

Figure 3. TEM images of an NW (a) prior to load, (b) under bending, (c) prior to and after fracture, as well as an (d) NW specimen with a fractured surface; (e) $I-V$ curves at two axially compressive stresses; (f) resistivity and conductivity; and (g) carrier concentration and mobility as a function of stress. Inset in (d) shows the enlarged TEM image of the fractured surface. Insets in (e) show the TEM images of an NW under stresses at 0.22 and $0.67 \mathrm{GPa}$ marked by black and red squares, respectively.

$\mathrm{NW}$, and $\varepsilon_{0}$ is the permittivity of free space. Carrier mobility, $\mu$, is calculated 26,30

$$
\mu=\frac{1}{n q \rho}
$$

where $n$ is the carrier concentration. In the calculations, $N_{\mathrm{d}}$ is the same as $n .{ }^{26} m_{\mathrm{r}}$ and $\varepsilon_{\mathrm{r}}$ for $3 \mathrm{C}$-SiC are $0.25^{31}$ and $9.72,{ }^{32}$ respectively. The carrier concentration is $3.46 \times 10^{17} \mathrm{~cm}^{-3}$ under compressive stress at $1.23 \mathrm{GPa}$. Resistivity and conductivity as a function of stress are illustrated in Figure 2(c). Carrier concentration and mobility under distinct compressive stress are depicted in Figure 2(d). Carrier concentration, mobility, and conductivity increases monotonically, whereas resistivity decreases with increasing compressive stress. To evaluate the piezoresistance effect of an NW, it is necessary to calculate the piezoresistance coefficient $P_{\mathcal{c}}$

$$
P_{c}=\frac{\rho-\rho_{0}}{\sigma \rho_{0}}
$$

where $\rho_{0}$ is the resistivity under zero stress, and $\rho$ is the resistivity under stress $\sigma$. To contact well between the flat W tip and NW in Figure S2, an axially smaller compressive force of $10 \mu \mathrm{N}$ is applied on the $\mathrm{W}$ tip. The measured $\sigma_{1}$ and $\rho_{1}$ are $0.21 \mathrm{GPa}$ and $2.11 \Omega \cdot \mathrm{cm}$, respectively. $P_{\mathrm{c}}$ recasts as

$$
P_{\mathrm{c}}=\frac{\rho-\rho_{1}}{\left(\sigma-\sigma_{1}\right) \rho_{1}}
$$

Piezoresistance coefficients $P_{c<111>}$ are $-48.89 \times 10^{-11}$ and $-87.81 \times 10^{-11} \mathrm{~Pa}^{-1}$ under axially compressive stress at 1.23 and $0.62 \mathrm{GPa}$, respectively, corresponding to carrier mobilities of 17.13 and $15.93 \mathrm{~cm}^{2} \mathrm{~V}^{-1} \mathrm{~s}^{-1}$. The $P_{\mathrm{c}<111>}$ of $-87.81 \times 10^{-11}$ $\mathrm{Pa}^{-1}$ and carrier mobility of $15.93 \mathrm{~cm}^{2} \mathrm{~V}^{-1} \mathrm{~s}^{-1}$ are 76.35 and 279.48 times those of $\mathrm{SiC}$ NWs under tensile strain, in which the $P_{\mathrm{c}<111>}$ and carrier mobility are $-1.15 \times 10^{-11} \mathrm{~Pa}^{-1}$ and $0.057 \mathrm{~cm}^{2} \mathrm{~V}^{-1} \mathrm{~s}^{-1}$, respectively. ${ }^{19}$ The piezoresistance coefficient and carrier mobility measured in this study are unprecedented, compared with previous reports. A relationship is presented between gauge factor (GF) and piezoresistance coefficient $^{19}$

$$
\mathrm{GF}=P_{\mathrm{c}} Y
$$

where $Y$ is the Young's modulus. The Young's modulus of SiC NW along the [111] orientation is $600 \mathrm{GPa}^{33}$ The piezoresistance coefficient of bulk $\mathrm{SiC}$ varies from $-2.12 \times$ $10^{-11}$ to $-5.3 \times 10^{-11} \mathrm{~Pa}^{-1}$. $^{34}$ In this work, the piezoresistance coefficient of NW is 16.56 times that of bulk SiC.

The NW devices are sometimes prepared and fabricated in vacuum. To investigate the piezoresistance effect of a clean surface, an NW was fractured under compression in the vacuum of the TEM, as shown in Figures 3(a-c). In Figure 3(c), there are two NWs present in an image. The two NWs are the same one prior to and after fracture because of the difference between the sample frequency of the camera in TEM and in situ TEM compression. In situ dynamic TEM compression of an NW is shown in Movies S4, S5, and S6 under compressive forces at 10,30 , and $32 \mu \mathrm{N}$, respectively. In the inset of Figure 3(d), the fracture is along the $\{111\}$ planes through cleavage. Accordingly, a clean surface of $\mathrm{SiC} \mathrm{NW}$ is obtained in vacuum. $I-V$ curves are shown in Figure 3(e) under axially compressive stress at 0.22 and $0.67 \mathrm{GPa}$. Nonlinear characteristics of $I-V$ curves confirm the Schottky contact in $\mathrm{W}-\mathrm{SiC}-\mathrm{Ag}$ connections, ${ }^{26,27}$ which is in good agreement with those in Figure 1(e). Nevertheless, $I-V$ curves in Figure 3(e) demonstrate an asymmetrical feature from forward to reverse bias regimes. This is distinct from that in Figure 1(e). It is because of that the NW in Figure 1(e) was exposed in air, resulting in impurities being absorbed and oxidization on the surface. However, the fractured surface in Figure 3(e) is fabricated in vacuum, absent of impurities and oxidation on the surface. Moreover, in Figure 3(e), one side is a clean surface fabricated in vacuum, and the other side absorbs impurities and oxidizes in air, leading to the asymmetrical characteristics of $I-V$ curves. Conductivity, carrier concentration, and mobility increase with stress; this is associated with a concomitant decrease in resistivity as depicted in Figure $3(\mathrm{f}, \mathrm{g})$. This is consistent with the results from Figures S2 (c,d). The piezoresistance coefficient $P_{c<111>}$ and carrier mobility in Figure 3(e) are $-94.78 \times 10^{-11} \mathrm{~Pa}^{-1}$ and $30.05 \mathrm{~cm}^{2} \mathrm{~V}^{-1} \mathrm{~s}^{-1}$ (Figure $3(\mathrm{~g})$ ), respectively, which are 
82.42 and 527.25 times those of NWs under tensile strain reported in previous literature. ${ }^{19}$ The unprecedented piezoresistance coefficient of the NW is 17.88 times that of bulk SiC. ${ }^{34}$ To confirm the unprecedented piezoresistance effect, photographs of a $\mathrm{SiC} \mathrm{NW}$ device prior to and after pressing are illustrated in Figure S3, and the dynamic pressing process is displayed in Movie S7. Prior to pressing, the conductance of the $\mathrm{SiC}$ NW device is low compared with the imposed voltage, resulting an unlit light (Figure S3(a)). After impressing, the conductance increases and the resistance decreases, leading to light (Figure S3(b)). High temperature to some materials can be considered a harsh environment. Si devices typically do not function at temperatures exceeding $200{ }^{\circ} \mathrm{C} .{ }^{8}$ The pressing was operated at $300{ }^{\circ} \mathrm{C}$, which is out of the scope of working conditions for normal Si devices. This demonstrates that the unprecedented piezoresistance effect of the $\mathrm{SiC} \mathrm{NW}$ device works well at high temperature.

To explore the mechanism of the unprecedented piezoresistance effect of $\mathrm{SiC} \mathrm{NW,} \mathrm{ab} \mathrm{initio} \mathrm{simulations} \mathrm{were} \mathrm{carried}$ out. A constructed crystalline model of $\mathrm{SiC} \mathrm{NW}$ is shown in Figure 4(a). Yellow, blue, and white balls represent Si, carbon,
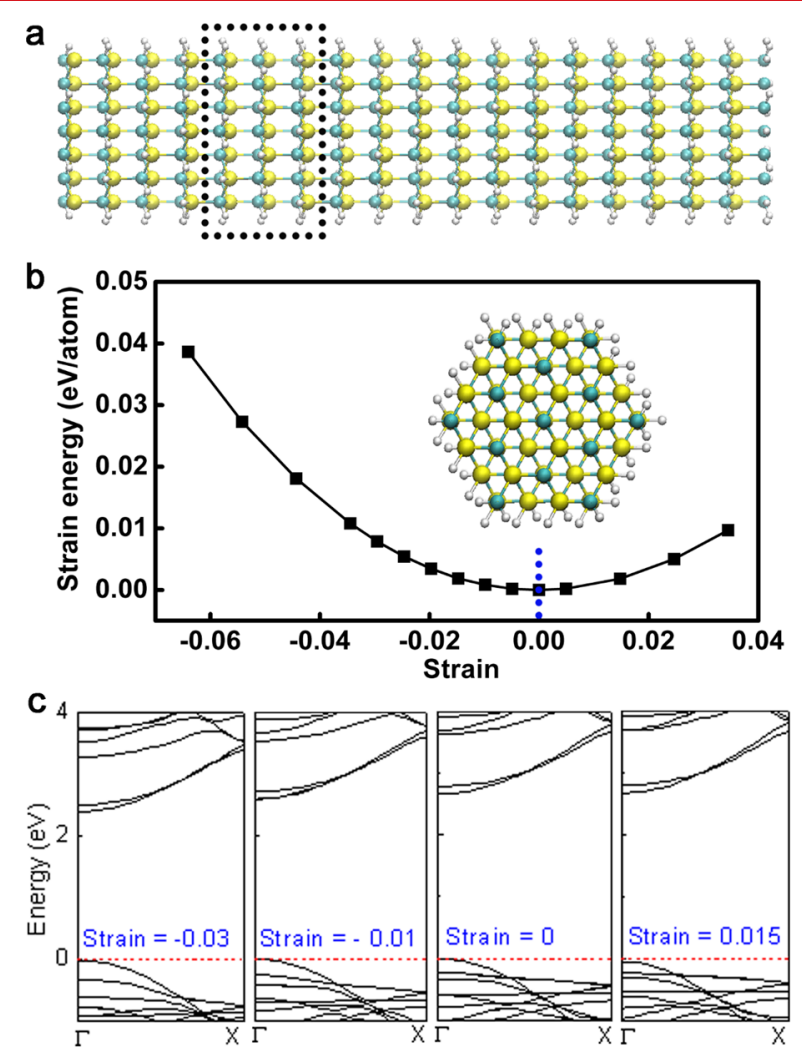

Figure 4. (a) Side view of the crystalline model of $\mathrm{SiC}$ NW used in ab initio simulations, (b) strain energy as a function of uniaxial strain, (c) band structure of the SiC NW at strains of $-0.03,-0.01,0$, and 0.015 . Inset in (b) shows the axial view of the NW in (a).

and hydrogen $(\mathrm{H})$ atoms, respectively. The axial direction of the NW is along the $\langle 111\rangle$ orientation, which agrees well with that in our experiment. A unit cell used in calculations is marked by a black dashed rectangle in Figure 4(a). The strain energy in Figure 4(b) reveals a parabolic shape, indicating that applied strain varying from -0.064 to 0.034 is in the elastic range. The representative electronic structure of the $\mathrm{SiC} \mathrm{NW}$ is illustrated in Figure 4(c). A direct band gap of $2.67 \mathrm{eV}$ is observed between the top of the valence band and the doubly generated conduction band minimum at the $\Gamma$ point. This is different from bulk $\mathrm{SiC}$, which has an indirect band gap of 2.39 $\mathrm{eV}^{35}$ Values of $\mathrm{SiC}$ NWs with smaller diameters are above $3.31 \mathrm{eV}^{36}$ because of quantum confinement. Hence, the simulation results are in good agreement with previous results. In Figure 4(c), the lowest conduction band steadily approaches the Fermi level with increasing compressive strain, leading to a narrower band gap. The energy band gap monotonically decreases with decreasing strain over the entire region from tensile to compressive strain, as depicted in Figure 5(a). The smaller band gap induces higher probability of charge carrier transport, which is consistent with higher conductivity as shown in Figures 2(c) and 3(f) under axial compression. Variation of the effective mass of charge carriers with strain is shown in Figure 5(b). Both conduction band bottom and valence band top have an effective mass of $0.44 \mathrm{~m}$. With applied axial strain, the effective mass reveals a negative correlation, on account of narrowing under compression and widening by tension of the band dispersion at the $\Gamma$ point. Band edge shifts of the conduction band bottom and valence band top are illustrated in Figures 5(c,d), respectively. Most remarkable is that the slope, $S$, of the band edge shift of the conduction band bottom decreases dramatically, approaching to approximately zero under compressive strain (Figure 5(c)). The carrier mobility, $\mu$, is estimated using the slope $S^{37,38}$

$$
\mu=\frac{2 \mathrm{q} \hbar^{3} E}{3 k T\left|m^{*}\right|^{2} S^{2}}
$$

where $E$ is the elastic modulus. As a consequence, carrier mobility will significantly increase under compressive strain, which is in good agreement with unprecedented carrier mobility in Figures $2(\mathrm{~d})$ and $3(\mathrm{~g})$. The synergistic effect between reduced band gap and increased carrier mobility under compressive strain considerably increases the transport properties of $\mathrm{SiC}$ NWs. This contributes greatly to the unprecedented piezoresistance effect observed in our experiments.

In conclusion, a new setup was developed to eliminate the contamination and damage induced by conventional depositions to fix NWs. In the setup, NWs were moved and transferred by a goat hair and then fixed by a conductive $\mathrm{Ag}$ epoxy glue in air. With the new fixing method, in situ TEM electromechanical measurements were conducted on the NWs. Piezoresistance coefficient and carrier mobility of a SiC NW are $-87.81 \times 10^{-11} \mathrm{~Pa}^{-1}$ and $15.93 \mathrm{~cm}^{2} \mathrm{~V}^{-1} \mathrm{~s}^{-1}$ under compressive stress respectively, which are 76.35 and 279.48 times those of $\mathrm{SiC}$ NWs previously reported. The unprecedented piezoresistance coefficient of $\mathrm{SiC} \mathrm{NW}$ is 16.56 times that of bulk SiC. An NW was fractured under axial compression in the TEM, and then, in situ TEM electromechanical measurements were performed. The piezoresistance coefficient and carrier mobility of the fractured SiC NW are 82.42 and 527.25 times those reported previously. The unprecedented piezoresistance coefficient of $\mathrm{SiC} \mathrm{NW}$ is 17.88 times that of its bulk counterparts. The outcomes found in this study pave a way to design and fabricate high performance semiconductor NW devices used in flexible electronics, NEMS, and nanoscale devices that could work at high temperature, high power, high frequency, and in harsh environments.

Experiments and Simulations. A TEM copper $(\mathrm{Cu})$ grid with a diameter of $3 \mathrm{~mm}$ was used to manipulate $\mathrm{SiC}$ NWs. ${ }^{38}$ 

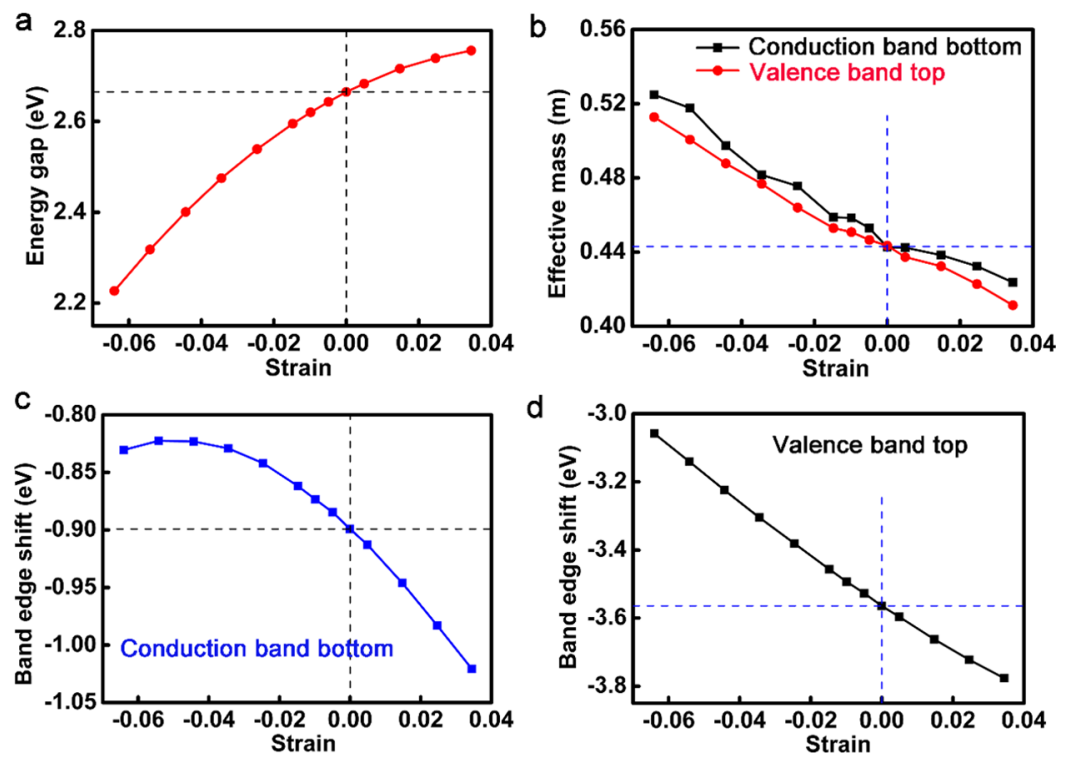

Figure 5. (a) Energy gap and (b) effective mass; band edge shift of (c) conduction band bottom and (d) valence band top as a function of strain.

A carbon film was covered on the $\mathrm{Cu}$ grid, which was removed by a fire ignited by a lighter. The $\mathrm{Cu}$ grid was then ultrasonically cleaned in acetone for $10 \mathrm{~min}$. After the $\mathrm{C}$ film was removed, the uniform holes with a diameter of $100 \mu \mathrm{m}$ on the $\mathrm{Cu}$ grid were exposed in air. NWs were put on the $\mathrm{Cu}$ grid, and they could be picked up using a goat hair through inserting the tapered tip into the hole and picking up the NW. This operation used the stiffness of the goat hair and attractive force between the goat hair and NW to overcome the force between the NW and $\mathrm{Cu}$ grid. Commercially available single crystal 3CSiC NWs (Nanjing XFNANO Materials Tech Co., Ltd., China) were used as specimens for the in situ TEM electromechanical measurements. NWs were characterized and measured by SEM (Verios G4 UC, Thermo Scientific, USA), TEM (Talos F200X, Themo Fisher Scientific, USA), and Raman spectra (Renishaw inVia Reflex, UK). They were ultrasonically dispersed in acetone solution for $3 \mathrm{~min}$, and then, the solution was absorbed by a pipet. A drop of acetone containing the NWs was dripped on the $\mathrm{Cu}$ grid. After evaporation of acetone, the NWs were dispersed on the $\mathrm{Cu}$ grid. A goat hair was taken from a Chinese writing brush (Figure S4(a)). It was glued by conductive Ag epoxy (CircuitWorks Conductive Epoxy, CW2400, Chemtronics, USA) on a toothpick. The opposite sharp end of the toothpick was inserted on one side of a polyethylene foam cuboid, and another side was glued by double faced adhesive tape on a glass block. The glass block was put on a mechanical stage of another optical microscope (NMM-800RF, Ningbo Yongxin Optics Co., Ltd., China). The goat hair was operated in 3D spaces at millimeters and micrometers by the mechanical stage. To manipulate NWs, a new setup was developed (Figure $\mathrm{S} 4(\mathrm{~b})$ ), in which the $\mathrm{Cu}$ grid was put on a mechanical stage of an optical microscope (Leica DM2500 M, Germany). The Cu grid on the mechanical stage was moved in three-dimensional (3D) space in millimeters and micrometers by coarse and fine focal adjustments. The goat hair had a tapered tip with diameters varied from several micrometers to hundreds of nanometers. An NW was picked up from the $\mathrm{Cu}$ grid by inserting the goat hair tip under the NW in a hole of the $\mathrm{Cu}$ grid (Figure S4(c)). After picking up, the $\mathrm{Cu}$ grid was replaced by a push-to-pull (PTP) device taken from an in situ TEM nanomechanical system (PI 95 PicoIndenter, Hysitron, Minneapolis, USA). The NW was manipulated by the goat hair in the developed setup to put it on one side of the siliconon-insulator (SOI) of the PTP device. It was fixed by conductive Ag epoxy onto the PTP (Figure S4(d)). To increase conductivity, a layer of conductive Ag paint was smeared on the surface of SOI. Then, the PTP device was mounted in the nanomechanical system. An in situ TEM system was inserted in a TEM (JEM-2100F, JEOL, Japan) to perform electromechanical coupling measurements. In situ TEM electromechanical measurements, mechanical force, and electrical signals were measured simultaneously. Meanwhile, the nanostructure of the NW was characterized and measured under strain. Mechanical force was applied by a load-controlled mode. The loading and unloading rates were $5 \mu \mathrm{N} / \mathrm{s}$, and the holding time was $20 \mathrm{~s}$. During holding, voltage was applied on the NW by the sweep mode. Voltages changed from -8 to $8 \mathrm{~V}$, and current was measured at the same time. In each $I-V$ curve, 160 points were measured, and each point lasted $50 \mathrm{~ms}$.

$\mathrm{Ab}$ initio simulations were performed to investigate the electronic properties under strain for the unprecedented piezoresistance effect using the Vienna $\mathrm{Ab}$ initio Simulation Package (VASP) code. $^{39,40}$ In the simulations, the generalized gradient approximation $^{41,42}$ was employed in Perdew-BurkeErnzerhof form for the exchange and correlation potential, which is together with the projector augmented wave (PAW) method. ${ }^{43}$ The constructed crystalline model consisted of 116 atoms, and periodic conditions were applied to the axial direction. The surface layer of the model was passivated by $\mathrm{H}$ atoms to eliminate the effect of dangling bonds. An energy cutoff of $400 \mathrm{eV}$ was chosen for the basis of the plane wave, and the model was fully relaxed when the forces on all atoms were less than $0.01 \mathrm{eV} / \AA$. The fully relaxed $\mathrm{SiC} \mathrm{NW}$ had a lattice constant of $7.644 \AA$ in the axial direction, and the lateral size was $13.4 \AA$. The axial lattice constant was changed to simulate the strain under axial force. ${ }^{44}$ 


\section{ASSOCIATED CONTENT}

Figure S1: SEM and TEM images as well as EDS and Raman spectra of SiC NWs; Figure S2: Schematic diagram of an in situ TEM electromechanical coupling measurement and TEM image of an NW prior to axial compression; Figure S3: Photographs of an $\mathrm{SiC} \mathrm{NW}$ device prior to and after pressing working at $300{ }^{\circ} \mathrm{C}$; Figure S4: Photographs of calligraphy written by a goat hair Chinese writing brush, a developed setup, optical image of a goat hair picking up an NW, and SEM image of an NW fixed by conductive Ag epoxy on a PTP device (PDF)

In situ dynamic TEM electromechanical measurements of an NW at $10 \mu \mathrm{N}$ (AVI)

In situ dynamic TEM electromechanical measurements of an $\mathrm{NW}$ at $30 \mu \mathrm{N}$ (AVI)

In situ dynamic TEM electromechanical measurements of an NW at $60 \mu \mathrm{N}$ (AVI)

In situ dynamic TEM compression of an NW under compressive force at $10 \mu \mathrm{N}$ (AVI)

In situ dynamic TEM compression of an NW under compressive force at $30 \mu \mathrm{N}$ (AVI)

In situ dynamic TEM compression of an NW under compressive force at $32 \mu \mathrm{N}$ (AVI)

Dynamic pressing process (AVI)

\section{AUTHOR INFORMATION}

\section{Corresponding Authors}

*E-mail: zzy@dlut.edu.cn. (Z.Y.Z.)

*E-mail: jiangnan@nimte.ac.cn. (N.J.)

*E-mail: tangchun@ujs.edu.cn. (C.T.)

\section{ORCID $\odot$}

Zhenyu Zhang: 0000-0002-2393-520X

\section{Author Contributions}

J.C. and Z.Z. contributed equally to this work.

\section{Author Contributions}

Z.Y.Z., N.J., and D.M.G. conceived the projects. Z.Y.Z. wrote the paper. J.F.C. designed and developed the operation method of NWs in air. J.F.C., D.D.L., D.L.Z., W.H., and H.H.L. performed the in situ TEM electromechanical measurements. C.T. conducted the ab initio simulations. Z.Y.Z., J.F.C., N.J., C.T., L.Z., C.Z., Y.L., and I.P.P. analyzed the mechanism of the unprecedented piezoresistance effect of $\mathrm{SiC}$ NWs under compressive strain. All authors discussed the results and commented on the manuscript.

\section{Notes}

The authors declare no competing financial interest.

\section{ACKNOWLEDGMENTS}

Z.Y.Z. appreciates the help for in situ TEM electromechanical measurements as well as kind discussions with Prof. Zhiwei Shan from Xi'an Jiaotong University and Director Lance Kuhn from Bruker Nano Surfaces. C.T. is grateful for valuable discussions with Prof. Changfeng Chen at University of Nevada Las Vegas. The authors acknowledge the financial support from the National Key R\&D Program of China (2018YFA0703400), the Excellent Young Scientists Fund of
NSFC (51422502), the Science Fund for Creative Research Groups of NSFC (51621064), the Changjiang Scholar Program of Chinese Ministry of Education, Program for Creative Talents in University of Liaoning Province (LR2016006), the Distinguished Young Scholars for Science and Technology of Dalian City (2016RJ05), the Xinghai Science Funds for Distinguished Young Scholars and Thousand Youth Talents at Dalian University of Technology, the Science Fund of State Key Laboratory of Tribology, Tsinghua University (SKLTKF17B19), the Science Fund of State Key Laboratory of Metastable Materials Science and Technology, Yanshan University (201813), and the Collaborative Innovation Center of Major Machine Manufacturing in Liaoning. Y.L. acknowledges the financial support from the QMUL-SBCS start up.

\section{REFERENCES}

(1) Banerjee, A.; Bernoulli, D.; Zhang, H. T.; Yuen, M. F.; Liu, J. B.; Dong, J. C.; Ding, F.; Lu, J.; Dao, M.; Zhang, W. J.; Lu, Y.; Suresh, S. Ultralarge Elastic Deformation of Nanoscale Diamond. Science 2018, 360, 300-302.

(2) He, R. R.; Yang, P. D. Giant Piezoresistance Effect in Silicon Nanowires. Nat. Nanotechnol. 2006, 1, 42-46.

(3) Winkler, K.; Bertagnolli, E.; Lugstein, A. Origin of Anomalous Piezoresistive Effects in VLS Grown Si Nanowires. Nano Lett. 2015, $15,1780-1785$.

(4) Hall, A. R.; Falvo, M. R.; Superfine, R.; Washburn, S. Electromechanical Response of Single-Walled Carbon Nanotubes to Torsional Strain in a Self-Contained Device. Nat. Nanotechnol. 2007, 2, 413-416.

(5) Greil, J.; Lugstein, A.; Zeiner, C.; Strasser, G.; Bertagnolli, E. Tuning the Electro-Optical Properties of Germanium Nanowires by Tensile Strain. Nano Lett. 2012, 12, 6230-6234.

(6) Zeng, L. J.; Gammer, C.; Ozdol, B.; Nordqvist, T.; Nygard, J.; Krogstrup, P.; Minor, A. M.; Jager, W.; Olsson, E. Correlation between Electrical Transport and Nanoscale Strain in InAs/ $\mathrm{In}_{0.6} \mathrm{Ga}_{0.4} \mathrm{As}$ Core-Shell Nanowires. Nano Lett. 2018, 18, 4949-4956.

(7) Li, X.; Wei, X. L.; Xu, T. T.; Pan, D.; Zhao, J. H.; Chen, Q. Remarkable and Crystal-Structure-Dependent Piezoelectric and Piezoresistive Effects of InAs Nanowires. Adv. Mater. 2015, 27, $2852-2858$

(8) Phan, H. P.; Dao, D. V.; Nakamura, K.; Dimitrijev, S.; Nguyen, N. T. The Piezoresistive Effect of SiC for MEMS Sensors at High Temperatures: A Review. J. Microelectromech. Syst. 2015, 24, 16631677.

(9) Melinon, P.; Masenelli, B.; Tournus, F.; Perez, A. Playing with Carbon and Silicon at the Nanoscale. Nat. Mater. 2007, 6, 479-490.

(10) Lee, T. H.; Bhunia, S.; Mehregany, M. Electromechanical Coupling at $500{ }^{\circ} \mathrm{C}$ with Silicon Carbide. Science 2010, 329, 13161318.

(11) Ishikawa, T.; Kohtoku, Y.; Kumagawa, K.; Yamamura, T.; Nagasawa, T. High-Strength Alkali-Resistant Sintered SiC Fibre Stable to $2200{ }^{\circ} \mathrm{C}$. Nature $1998,391,773-775$.

(12) Nakamura, D.; Gunjishima, I.; Yamaguchi, S.; Ito, T.; Okamoto, A.; Kondo, H.; Onda, S.; Takatori, K. Ultrahigh-Quality Silicon Carbide Single Crystals. Nature 2004, 430, 1009-1012.

(13) Han, X. D.; Zhang, Y. F.; Zheng, K.; Zhang, X. N.; Zhang, Z.; Hao, Y. J.; Guo, X. Y.; Yuan, J.; Wang, Z. L. Low-Temperature In Situ Large Strain Plasticity of Ceramic SiC Nanowires and its AtomicScale Mechanism. Nano Lett. 2007, 7, 452-457.

(14) Widmann, M.; Lee, S. Y.; Rendler, T.; Son, N. T.; Fedder, H.; Paik, S.; Yang, L. P.; Zhao, N.; Yang, S.; Booker, I.; Denisenko, A.; Jamali, M.; Momenzadeh, S. A.; Gerhardt, I.; Ohshima, T.; Gali, A.; Janzen, E.; Wrachtrup, J. Coherent Control of Single Spins in Silicon Carbide at Room Temperature. Nat. Mater. 2015, 14, 164-168. 
(15) Lugstein, A.; Steinmair, M.; Steiger, A.; Kosina, H.; Bertagnolli, E. Anomalous Piezoresistance Effect in Ultrastrained Silicon Nanowires. Nano Lett. 2010, 10, 3204-3208.

(16) Koehl, W. F.; Buckley, B. B.; Heremans, F. J.; Calusine, G.; Awschalom, D. D. Room Temperature Coherent Control of Defect Spin Qubits in Silicon Carbide. Nature 2011, 479, 84-87.

(17) Kim, J.; Park, H.; Hannon, J. B.; Bedell, S. W.; Fogel, K.; Sadana, D. K.; Dimitrakopoulos, C. Layer-Resolved Graphene Transfer via Engineered Strain Layers. Science 2013, 342, 833-836.

(18) Emtsev, K. V.; Bostwick, A.; Horn, K.; Jobst, J.; Kellogg, G. L.; Ley, L.; McChesney, J. L.; Ohta, T.; Reshanov, S. A.; Rohrl, J.; Rotenberg, E.; Schmid, A. K.; Waldmann, D.; Weber, H. B.; Seyller, T. Towards Wafer-Size Graphene Layers by Atmospheric Pressure Graphitization of Silicon Carbide. Nat. Mater. 2009, 8, 203-207.

(19) Shao, R. W.; Zheng, K.; Zhang, Y. F.; Li, Y. J.; Zhang, Z.; Han, $\mathrm{X}$. D. Piezoresistance Behaviors of Ultra-Strained SiC Nanowires. Appl. Phys. Lett. 2012, 101, 233109.

(20) Zeng, H. J.; Li, T.; Bartenwerfer, M.; Fatikow, S.; Wang, Y. L. In Situ SEM Electromechanical Characterization of Nanowire using an Electrostatic Tensile Device. J. Phys. D: Appl. Phys. 2013, 46, 305501.

(21) Wang, X. G.; Chen, K.; Zhang, Y. Q.; Wan, J. C.; Warren, O. L.; Oh, J.; Li, J.; Ma, E.; Shan, Z. W. Growth Conditions Control the Elastic and Electrical Properties of $\mathrm{ZnO}$ Nanowires. Nano Lett. 2015, $15,7886-7892$.

(22) Bernal, R. A.; Filleter, T.; Connell, J. G.; Sohn, K.; Huang, J. X.; Lauhon, L. J.; Espinosa, H. D. In Situ Electron Microscopy Four-Point Electromechanical Characterization of Freestanding Metallic and Semiconducting Nanowires. Small 2014, 10, 725-733.

(23) Gao, D.; Yang, Z. Y.; Zheng, L. L.; Zheng, K. Piezoresistive Effect of N-Type $<111>$-Oriented Si Nanowires under Large Tension/Compression. Nanotechnology 2017, 28, 095702.

(24) Zhang, Y. F.; Han, X. D.; Zheng, K.; Zhang, Z.; Zhang, X. N.; Fu, J. Y.; Ji, Y.; Hao, Y. J.; Guo, X. Y.; Wang, Z. L. Direct Observation of Super-Plasticity of Beta-SiC Nanowires at Low Temperature. Adv. Funct. Mater. 2007, 17, 3435-3440.

(25) Dai, S.; Zhao, J.; He, M.; Wang, X. G.; Wan, J. C.; Shan, Z. W.; Zhu, J. Elastic Properties of GaN Nanowires: Revealing the Influence of Planar Defects on Young's Modulus at Nanoscale. Nano Lett. 2015, $15,8-15$.

(26) Zhang, Z. Y.; Yao, K.; Liu, Y.; Jin, C. H.; Liang, X. L.; Chen, Q.; Peng, L. M. Quantitative Analysis of Current-Voltage Characteristics of Semiconducting Nanowires: Decoupling of Contact Effects. Adv. Funct. Mater. 2007, 17, 2478-2489.

(27) Rhoderick, E. H.; Williams, R. H. Metal-Semiconductor Contacts, 2nd ed.; Oxford University Press, 1988.

(28) Chou, X. J.; Zhu, J.; Qian, S.; Niu, X. S.; Qian, J. C.; Hou, X. J.; Mu, J. L.; Geng, W. P.; Cho, J. D.; He, J.; Xue, C. Y. All-in-One FillerElastomer-Based High-Performance Stretchable Piezoelectric Nanogenerator for Kinetic Energy Harvesting and Self-Powered Motion Monitoring. Nano Energy 2018, 53, 550-558.

(29) He, J.; Wen, T.; Qian, S.; Zhang, Z. X.; Tian, Z. M.; Zhu, J.; Mu, J. L.; Hou, X. J.; Geng, W. P.; Cho, J. D.; Han, J. Q.; Chou, X. J.; Xue, C. Y. Triboelectric-Piezoelectric-Electromagnetic Hybrid Nanogenerator for High-Efficient Vibration Energy Harvesting and SelfPowered Wireless Monitoring System. Nano Energy 2018, 43, 326339.

(30) Zhang, Z. Y.; Jin, C. H.; Liang, X. L.; Chen, Q.; Peng, L. M. Current-Voltage Characteristics and Parameter Retrieval of Semiconducting Nanowires. Appl. Phys. Lett. 2006, 88, 073102.

(31) Kaplan, R.; Wagner, R. J.; Kim, H. J.; Davis, R. F. Electron Cyclotron Resonance in Cubic SiC. Solid State Commun. 1985, 55, 67-69.

(32) Patrick, L.; Choyke, W. J. Static Dielectric Constant of SiC. Phys. Rev. B 1970, 2, 2255-2256.

(33) Wong, E. W.; Sheehan, P. E.; Lieber, C. M. Nanobeam Mechanics: Elasticity, Strength, and Toughness of Nanorods and Nanotubes. Science 1997, 277, 1971-1975.
(34) Shor, J. S.; Goldstein, D.; Kurtz, A. D. Characterization of NType $\beta$-SiC as a Piezoresistor. IEEE Trans. Electron Devices 1993, 40, $1093-1099$.

(35) Harris, G. L. Properties of Silicon Carbide; INSPEC, the Institution of Electrical Engineers: London, United Kingdom, 1995.

(36) Yan, B. H.; Zhou, G.; Duan, W. H.; Wu, J.; Gu, B. L. UniaxialStress Effects on Electronic Properties of Silicon Carbide Nanowires. Appl. Phys. Lett. 2006, 89, 023104.

(37) Kou, L. Z.; Ma, Y. D.; Zhou, L. J.; Sun, Z. Q.; Gu, Y. T.; Du, A. J.; Smith, S.; Chen, C. F. High-Mobility Anisotropic Transport in Few-Layer Gamma-B-28 Films. Nanoscale 2016, 8, 20111-20117.

(38) Sharma, S. K.; Manoj Kumar, B. V.; Kim, Y. W. Tribology of WC Reinforced SiC Ceramics: Influence of Counterbody. Friction 2019, 7, 129-142.

(39) Kresse, G.; Furthmuller, J. Efficient Iterative Schemes for $A b$ Initio Total-Energy Calculations using a Plane-Wave Basis Set. Phys. Rev. B: Condens. Matter Mater. Phys. 1996, 54, 11169-11186.

(40) Kim, T. H.; Puggioni, D.; Yuan, Y.; Xie, L.; Zhou, H.; Campbell, N.; Ryan, P. J.; Choi, Y.; Kim, J. W.; Patzner, J. R.; Ryu, S.; Podkaminer, J. P.; Irwin, J.; Ma, Y.; Fennie, C. J.; Rzchowski, M. S.; Pan, X. Q.; Gopalan, V.; Rondinelli, J. M.; Eom, C. B. Polar Metals by Geometric Design. Nature 2016, 533, 68-72.

(41) Perdew, J. P.; Burke, K.; Ernzerhof, M. Generalized Gradient Approximation Made Simple. Phys. Rev. Lett. 1996, 77, 3865-3868.

(42) Carrez, P.; Ferre, D.; Cordier, P. Implications for Plastic Flow in the Deep Mantle from Modelling Dislocations in $\mathrm{MgSiO}_{3}$ Minerals. Nature 2007, 446, 68-70.

(43) Bediako, D. K.; Rezaee, M.; Yoo, H.; Larson, D. T.; Zhao, S. Y. F.; Taniguchi, T.; Watanabe, K.; Brower-Thomas, T. L.; Kaxiras, E.; Kim, P. Heterointerface Effects in the Electrointercalation of Van Der Waals Heterostructures. Nature 2018, 558, 425-429.

(44) Ewen, J. P.; Heyes, D. M.; Dini, D. Advances in Nonequilibrium Molecular Dynamics Simulations of Lubricants and Additives. Friction 2018, 6, 349-386. 